\title{
Tipologías de consumidores según el estilo de vida en relación a la alimentación: un estudio exploratorio en el sur de Chile
}

\author{
Consumer typology according \\ to food-related lifestyle: \\ an exploratory study in southern Chile
}

\begin{abstract}
With the aim of characterizing consumer typologies on the basis of food-related lifestyle in an exploratory manner, a survey was applied to a sample of 316 people in the main cities of southern Chile. The instruments for collecting data included an adaptation of the Food-related Lifestyle (FRL) questionnaire and the Satisfaction with Food-related Life (SWFL) scale. Three main typologies were distinguished using a cluster analysis. The majority (30.1\%) preferred easily prepared food, planned their diet and maintained their eating habits. The second group (17.4\%) enjoyed eating out and dinners with friends at home, but they preferred known foods, and were programmed and constant in their eating habits. The third typology (14.6\%) enjoyed sharing a meal with friends, but did not like cooking or using easy-to-prepare foods. The typologies displayed different profiles based on demographics. They differed in the food consumption frequency in the home and in the frequency of eating out. Differences were observed in the restriction of meals and dressings for health reasons and in the satisfaction with their food-related life.
\end{abstract}

Key words: Food-related lifestyle, satisfaction with food-related Life, eating habits.

\section{INTRODUCCIÓN}

La situación alimentaria de los países de América Latina está relacionada con los cambios sociodemográficos, económicos, dietarios y en los estilos de vida que ha tenido la población. En Chile estos cambios han ocurrido aceleradamente en las últimas décadas, lo que se ha traducido en un aumento en el consumo de alimentos ricos en colesterol, grasas saturadas, azúcar y sodio, entre otros, trayendo como consecuencia altas prevalencias de obesidad y de enfermedades crónicas no transmisibles (1). A consecuencia de la occidentalización de la dieta, en Chile el consumo de grasa ha aumentado con rapidez, mientras el consumo de frutas y verduras es bajo (2). Tomando en cuenta que, además, aproximadamente el $90 \%$ de la población es sedentaria, la prevalencia de sobrepeso y obesidad en el país ha alcanzado cifras hasta $66,7 \%$ (39,3\%
Berta Schnettler M. (1) Marcos Mora G. (2) Nataly Mills Q. (1) Horacio Miranda V. (1) José Sepúlveda M. (3) Marianela Denegri C. (3) Germán Lobos A. (4)

(1) Departamento de Producción Agropecuaria, Facultad de Ciencias Agropecuarias y Forestales, Universidad de La Frontera, Temuco, Chile. (2) Departamento de Economía Agraria, Facultad de Ciencias Agronómicas, Universidad de Chile, Santiago, Chile. (3) Departamento de Psicología, Facultad de Educación y Humanidades. Universidad de La Frontera, Temuco, Chile. (4) Escuela de Ingeniería Comercial, Facultad de Ciencias Empresariales, Universidad de Talca, Talca, Chile.

Dirigir la correspondencia a: Dra. Berta Schnettler M. Profesor Asociado

Departamento de Producción Agropecuaria Facultad de Ciencias Agropecuarias y Forestales Universidad de la Frontera Casilla 54-D Temuco, Chile Teléfonos: 56-45-325655/56-45-325458 E-mail: bschnett@ufro.c.

Este trabajo fue recibido el 12 de Junio de 2012 y aceptado para ser publicado el 20 de Septiembre de 2012.

sobrepeso, $25,1 \%$ obesidad y $2,3 \%$ obesidad mórbida) (3).

El estilo de vida alude a la forma de vivir de las personas y se interrelaciona estrechamente con los componentes motivacionales y conductuales; influenciado por costumbres, hábitos, modas y valores (4). El estilo de vida incluye patrones de conducta, individuales y colectivos, que demuestran cierta consistencia en el tiempo, bajo condiciones más o menos constantes, y pueden constituirse en dimensiones de riesgo o de seguridad dependiendo de su naturaleza (5).

Cuando el campo se delimita a la salud, las pruebas empíricas acumuladas en el tema permiten afirmar que son las conductas las que tienen un impacto muy importante en la salud, en la medida que, su presencia o ausencia, puede constituir un factor de riesgo o de protección para el individuo (6). Dentro de los patrones de comportamiento que confor- 
man los estilos de vida se incluyen prácticas saludables y no saludables que interactúan entre sí (7).

Las personas que tienen comportamientos no saludables estables en el tiempo, como el desequilibrio en la dieta, tienen una mayor probabilidad de desarrollar enfermedades, comparadas con aquellas personas que no incluyen estas prácticas en su estilo de vida (8). Los estilos de vida saludables propician la adquisición y mantenimiento en la cotidianidad de pautas de conducta que de manera individual y colectiva mejoran la calidad de vida; incluye patrones de conducta, creencias, conocimientos, hábitos y acciones de las personas para mantener, restablecer o mejorar su salud, bienestar y calidad de vida (9).

Existe evidencia que asocia el bienestar de las personas con su alimentación y con las preferencias hacia alimentos (10). Asimismo, estudios recientes relacionan la satisfacción con la alimentación con los hábitos alimentarios $(10,11)$ y con un buen estado de salud (12).

La investigación del estilo de vida de las personas tiene una larga trayectoria. Inicialmente se utilizó en forma general para medir las actitudes, intereses y opiniones, como base para segmentarlos. Más adelante, se introdujo la noción de estilos de vida en dominios específicos. El cuestionario de estilos de vida en relación a la alimentación (FRL por sus siglas en inglés: Food-related Lifestyle) fue introducido en la literatura a mediados de la década de los 90 (13), Ilegando a ser el instrumento más utilizado para distinguir tipologías de personas en el dominio de la alimentación (14-16). El cuestionario FRL intenta caracterizar a las personas según sus hábitos de preparación y consumo de alimentos y a los valores que guían su vida. Desde su introducción el FRL ha sido utilizado en numerosos países desarrollados, demostrando su utilidad para explicar la elección de ciertos alimentos (14-17), y como potencial predictor de obesidad en Europa (18).

El objetivo de esta investigación de tipo exploratoria fue distinguir tipologías de personas en base a su estilo de vida en relación a la alimentación en las principales ciudades del sur del Chile, junto con caracterizarlas en términos sociodemográficos, de hábitos de consumo de alimentos fuera y dentro del hogar y según la satisfacción con su alimentación.

\section{SUJETOS Y MÉTODO}

El presente estudio de tipo exploratorio corresponde a los resultados obtenidos en el primer año de ejecución del Proyecto Fondecyt 1100611. Se aplicó un cuestionario estructurado en forma personal a una muestra de 316 personas. Como criterios de inclusión en la muestra se consideró personas de ambos géneros, mayores de 18 años (sin límite superior de edad), que fueran responsables de la compra de alimentos para su hogar. El tamaño de la muestra se obtuvo mediante el procedimiento de muestreo aleatorio estratificado-variable dicotómica según la población de las ciudades con más de 100.000 habitantes ubicadas entre Las regiones de Arica y Parinacota y la Región de Los Lagos al Censo de 2002 (19), considerando $95 \%$ de confianza, 3\% de error de estimación con p y q de 0,5 (20). De esta forma se estimó una muestra de 1.852 personas distribuidas mediante afijación proporcional en las principales ciudades de estas regiones. Por operatividad en el proceso de recolección de la información, este tamaño muestral fue subdividido en zonas según la planificación de actividades del proyecto Fondecyt 1100611, que consideró comenzar con la aplicación de encuestas en la zona sur que comprendió las Regiones del Biobío, La Araucanía y Los Lagos. De esta forma, los resultados de tipo exploratorio que se presentan en este estudio corresponden a las encuestas reali- zadas en las ciudades con más de 100.000 habitantes de estas regiones: Chillán (47 encuestas), Concepción (149 encuestas), Temuco (72 encuestas) y Puerto Montt (48 encuestas).

\section{Instrumento}

Como instrumento de recogida de información se utilizó un cuestionario estructurado con dos escalas. La primera correspondió a una adaptación del cuestionario de estilos de vida en relación a la alimentación (FRL) propuesto inicialmente por Brunsø y Grunert (13) con 69 ítems. De éstos se eligieron, en forma exploratoria, aquellos más pertinentes a la realidad alimentaria en Chile, quedando constituido por 26 ítems. El encuestado debió responder su grado de acuerdo con cada uno de los ítems mediante una escala tipo Likert de 7 niveles (1: completamente en desacuerdo, 7: completamente de acuerdo).

La segunda correspondió a la escala SWFL (Satisfaction with Food-related Life), que fue propuesta y probada en Europa por Grunert et al. (21) mostrando adecuados niveles de consistencia interna $(0,71-0,89)$ y sólo una dimensión que agrupa los cinco ítems de la escala. El encuestado debió responder su grado de acuerdo con cada ítem mediante una escala tipo Likert de 6 niveles (1: completamente en desacuerdo, 6: completamente de acuerdo).

A continuación, se consultó la frecuencia de consumo en el hogar de 10 grupos de alimentos y bebidas tomadas de la Encuesta de Presupuestos Familiares del Instituto Nacional de Estadísticas (22). Luego se preguntó la frecuencia de comidas fuera del hogar y de consumo de comidas preparadas. Adicionalmente, se consultó si la persona encuestada o algún integrante de su familia restringe el consumo de alguno de los siguientes alimentos por motivos de salud o prevención: azúcar, productos de repostería, sal, grasas y frituras; pastas, arroz o papas; carnes rojas, café y alcohol. Se incluyeron preguntas de clasificación de los encuestados: género, edad, estado civil, número de integrantes de la familia, presencia de hijos en el hogar y sus edades, zona de residencia, ocupación y estudios del jefe de hogar y, la tenencia de 10 bienes domésticos. Estas dos últimas variables permiten determinar el nivel socioeconómico según Adimark (23), correspondiente a ABC1 (alto y medio-alto), C2 (medio-medio), C3 (medio-bajo), D (bajo), y E (muy bajo).

La encuesta fue aplicada por un encuestador previamente entrenado (psicólogo) en centros comerciales con supermercado y patio de comida, ubicados en distintas zonas socioeconómicas de las ciudades en estudio, entre julio y septiembre de 2010. El encuestador interceptó personas que iban saliendo de los supermercados y de los patios de comida, les explicó los objetivos de la encuesta, el manejo de la información obtenida en la encuesta con estricta confidencialidad y, a continuación, les preguntó si estaban dispuestos a responder el cuestionario. Los participantes que aceptaron responder la encuesta firmaron consentimientos informados previo a la aplicación del cuestionario. El cuestionario fue validado previamente con un pretest con el $5 \%$ de la muestra.

\section{Análisis estadístico}

El análisis de los resultados fue realizado con el programa SPSS 16.0 en español para Windows. La extracción de factores de las escalas se realizó con análisis factorial (AF) de componentes principales, considerando autovalores mayores que 1. Para el FRL se utilizó rotación de factores Varimax con Kaiser. La consistencia interna de las escalas y de sus componentes fue calculada usando el coeficiente $\alpha$ de Cronbach. De esta 
forma, mediante análisis factorial de componentes principales se obtuvieron cinco dimensiones que permitieron caracterizar las relaciones entre 16 de los 26 ítems utilizados del FRL, con el 63,3\% de la varianza explicada. El coeficiente a Cronbach obtenido en forma global por el FRL $(0,928)$ permite concluir que el instrumento es fiable (24). Los coeficientes $\alpha$ Cronbach por componente se encuentran dentro de los rangos reportados en estudios previos $(0,533$ y 0,847) (18) (tabla 1). En esta investigación la SWFL tuvo un a Cronbach de 0,993 y un factor que agrupó los cinco ítems con el 76,6\% de la varianza explicada. El 28,8\% de la muestra resultó extremadamente satisfecho; $34,8 \%$ satisfecho; $22,2 \%$ medianamente satisfecho y $14,4 \%$ insatisfecho con su alimentación.

Para determinar tipologías de consumidores según los resultados del FRL, se usó análisis cluster de conglomerados jerárquicos, con el método de Ward como forma de encadenamiento y la distancia euclídea al cuadrado como medida de similitud entre objetos. Este análisis se realizó sobre los Z-scores resultantes del AF realizado al FRL. El número de grupos se obtuvo mediante la determinación del porcentaje de cambio de los coeficientes de conglomeración recompuestos. Para describir las tipologías se aplicó test de Chi2 para las variables discretas, y análisis de varianza de un factor a los Z-scores obtenidos del AF. Debido que el estadístico de Levenne mostró varianzas no homogéneas en las variables continuas, aquellas cuyo análisis de varianza indicó diferencias significativas $(p \leq 0,001)$, fueron sometidas a la Prueba de Comparaciones Múltiples de T3 de Dunnett (24).

La realización del estudio fue aprobada por el Comité de Bioética de la Facultad de Ciencias Agropecuarias y Forestales de la Universidad de La Frontera.

\section{RESULTADOS}

La muestra estuvo compuesta en mayor proporción por mujeres (53,5\%), de 45 años y menos (70,5\%); solteros, separados, viudos y divorciados $(52,2 \%)$, de grupos familiares formados por tres a cuatro integrantes $(53,5 \%)$, sin presencia de hijos en el hogar $(37,7 \%)$ y con hijos entre 5 y 17 años

\section{TABLA 1}

Resultados del análisis factorial de componentes principales para el $\mathrm{FRL}$, en las principales ciudades del sur de Chile, septiembre de 2010.

Ítem del FRL

Le gusta cocinar.

Es un excelente cocinero/a.

Ser reconocido por sus habilidades de cocina, es muy importante para su auto-estima.

Busca formas nuevas de preparar comidas.

Disfruta de comer en restaurantes con familia y amigos (No lo considera un lujo).

Salir a comer fuera es parte de sus hábitos alimenticios regulares.

Le gusta compartir con amigos disfrutando de una comida en el hogar.

Las recetas ya conocidas, son las mejores.

Sólo compra alimentos que le son familiares.

Un plato familiar le da una sensación de seguridad.

Usa bastantes alimentos precocinados en su cocina.

Usa bastantes mezclas preparadas, por ejemplo masas para hornear y sopas instantáneas.

Los alimentos congelados son una parte importante de su despensa (usa productos congelados en su cocina).

Lo que van a cenar se planifica generalmente durante la hora de almuerzo.

Planifica lo que van a comer, con un par de días de anticipación,

Le agrada mantener sus hábitos alimentarios

Varianza explicada por componente (\%)

$\alpha$ Cronbach por componente
1

0,867

0,857

0,689

0,070

Componente1
3

2

4

5

$\begin{array}{lllll}0,630 & 0,306 & -0,004 & -0,085 & 0,265 \\ 0,036 & 0,870 & -0,016 & 0,048 & -0,052\end{array}$

0,02

0,746

$-0,032$

0,279

0,012

0,186

0,682

0,155

$-0,049$

0,071

$-0,063$

$-0,026$

0,846

0,141

$-0,004$

0,038

$-0,033$

0,808

0,064

$-0,046$

0,056

0,208

0,689

0,033

0,264

$-0,039$

$-0,009$

0,053

0,793

0,062

0,045

0,038

0,087

0,735

$-0,026$

0,204

0,085

0,685

0,067

$\begin{array}{ccccc}0,013 & 0,155 & 0,129 & 0,182 & 0,777 \\ 0,218 & 0,085 & 0,080 & 0,274 & 0,696 \\ 0,151 & 0,102 & 0,214 & 0,073 & 0,639 \\ 15,6 & 12,6 & 12,3 & 12,0 & 10,8 \\ 0,790 & 0,701 & 0,715 & 0,705 & 0,700\end{array}$

Método de extracción: Análisis de componentes principales, Método de rotación: Normalización Varimax con Kaiser. La rotación ha convergido en 5 iteraciones. Medida de adecuación muestral Keiser-Meyer-Olkin $(K M O)=0,703$. Prueba de esfericidad de Bartlett, Chi-cuadrado aproximado = 1318,$163 ; g I=120 ; p=0,000$.

${ }^{1}$ Componente 1: Gusto por cocinar. Componente 2: Vida social. Componente 3: Tradición. Componente 4: Conveniencia. Componente 5: Planificación. 
$(46,2 \%)$, residentes en zonas urbanas $(98,8 \%)$; con estudios medios completos $(30,1 \%)$ y universitarios completos o más $(32,6 \%)$, empleados públicos y particulares $(64,6 \%)$, de los niveles socioeconómicos alto y medio-alto $(42,1 \%)$ y mediomedio (34,2\%), no mapuche (94,9\%). Mediante análisis clúster se distinguieron siete tipologías con distinto perfil (tabla 2) de género, estado civil, tamaño familiar, presencia y edad de hijos y en el nivel de satisfacción con la alimentación $(p \leq 0,05)$. Las tipologías se diferenciaron significativamente en los Z-scores (tabla 2) de los cinco componentes obtenidos del FRL $(p \leq 0,001)$. Estas difirieron en la frecuencia de consumo (tabla 4$)$ de carne, pescados y mariscos $(p \leq 0,001)$, cereales y pastas, verduras y tubérculos $y$, bebidas y jugos $(p \leq 0,05)$. Se diferenciaron en la frecuencia de comidas en restaurantes, locales de comida rápida, comida preparada $(p \leq 0,001)$ y en puestos ambulantes $(p \leq 0,05)$, junto con diferencias en la restricción de comidas por motivos de salud: pastas, arroz y papas; carnes rojas ( $p \leq 0,001)$, azúcar, productos de repostería, sal y café $(p \leq 0,05)$ (tabla 5$)$. Las características de las tipologías se describen a continuación:

El grupo 1 (11,1\% de la muestra) estuvo compuesto por mayor proporción de personas solteras, separadas, viudas y divorciadas $(71,4 \%)$, familias con tres a cuatro integrantes (71,4\%), sin hijos $(57,1 \%)$ y menor presencia de personas ex-

TABLA 2

Características sociodemográficas y nivel de satisfacción con la alimentación (\%) de los grupos obtenidos a través de análisis de clúster, en las principales ciudades del sur de Chile, septiembre de 2010.

\begin{tabular}{|c|c|c|c|c|c|c|c|c|}
\hline & Grupo 1 & Grupo 2 & Grupo 3 & Grupo 4 & Grupo 5 & Grupo 6 & Grupo 7 & $\mathrm{P}$ \\
\hline \multicolumn{9}{|l|}{ Género } \\
\hline Masculino & 37,1 & 37,9 & 50,0 & 79,2 & 54,2 & 38,2 & 59,5 & \multirow[t]{2}{*}{0,004} \\
\hline Femenino & 62,9 & 62,1 & 50,0 & 20,8 & 45,8 & 61,8 & 40,5 & \\
\hline \multicolumn{9}{|l|}{ Estado civil } \\
\hline Solteros, separados, viudos, divorciados & 71,4 & 44,2 & 52,2 & 70,8 & 45,8 & 54,5 & 43,2 & \multirow[t]{2}{*}{0,049} \\
\hline Casados o viven en pareja & 28,6 & 55,8 & 47,8 & 29,2 & 54,2 & 45,5 & 56,8 & \\
\hline \multicolumn{9}{|l|}{ Tamaño del grupo familiar } \\
\hline $1-2$ integrantes & 20,0 & 8,4 & 8,7 & 4,2 & 20,8 & 12,7 & 18,9 & \multirow[t]{3}{*}{0,007} \\
\hline 3-4 integrantes & 71,4 & 55,8 & 45,7 & 33,3 & 50,0 & 52,7 & 56,8 & \\
\hline 5 o más integrantes en la familia & 8,6 & 35,8 & 45,7 & 62,5 & 29,2 & 34,5 & 24,3 & \\
\hline \multicolumn{9}{|l|}{ Presencia y edad de hijos en el hogar } \\
\hline Sin niños en el hogar & 57,1 & 28,4 & 43,5 & 20,8 & 33,3 & 43,6 & 40,5 & \multirow[t]{4}{*}{0,008} \\
\hline Niños menores de 5 años & 20,0 & 25,3 & 13,0 & 8,3 & 4,2 & 7,3 & 18,9 & \\
\hline Niños entre 5 y 13 años & 14,3 & 27,4 & 26,1 & 45,8 & 20,8 & 25,5 & 16,2 & \\
\hline Niños entre 14 y 17 años & 8,6 & 18,9 & 17,4 & 25,0 & 41,7 & 23,6 & 24,3 & \\
\hline \multicolumn{9}{|l|}{ Satisfacción con la alimentación } \\
\hline Insatisfecho & 22,9 & 10,5 & 13,0 & 41,7 & 12,5 & 3,6 & 16,2 & \multirow[t]{4}{*}{0,002} \\
\hline Medianamente satisfecho & 31,4 & 22,1 & 13,0 & 20,8 & 25,0 & 18,2 & 29,7 & \\
\hline Satisfecho & 34,3 & 40,0 & 39,1 & 25,0 & 33,3 & 30,9 & 29,7 & \\
\hline Extremadamente satisfecho & 11,4 & 27,4 & 34,8 & 12,5 & 29,2 & 47,3 & 24,3 & \\
\hline
\end{tabular}

Valores de P obtenidos con la Prueba $\mathrm{Chi}^{2}$

\section{TABLA 3}

Promedios de valores de puntaje $\mathbf{Z}$ de los grupos obtenidos a través de análisis de clúster, en las principales ciudades del sur de Chile, septiembre de 2010.

\begin{tabular}{lccccccccc}
\hline Componente & $\begin{array}{c}\text { Grupo 1 } \\
(\mathrm{n}=35)\end{array}$ & $\begin{array}{c}\text { Grupo 2 } \\
(\mathrm{n}=95)\end{array}$ & $\begin{array}{c}\text { Grupo 3 } \\
(\mathrm{n}=46)\end{array}$ & $\begin{array}{c}\text { Grupo 4 } \\
(\mathrm{n}=24)\end{array}$ & $\begin{array}{c}\text { Grupo 5 } \\
(\mathrm{n}=24)\end{array}$ & $\begin{array}{c}\text { Grupo 6 } \\
(\mathrm{n}=55)\end{array}$ & $\begin{array}{c}\text { Grupo } 7 \\
(\mathrm{n}=37)\end{array}$ & $\mathrm{F}$ & P-value \\
1. Gusto por cocinar & $-0,19 \mathrm{~b}$ & $0,07 \mathrm{~b}$ & $-1,22 \mathrm{c}$ & $0,40 \mathrm{~b}$ & $1,22 \mathrm{a}$ & $0,44 \mathrm{~b}$ & $-0,19 \mathrm{~b}$ & 32,348 & $0,000 *$ \\
2. Vida social & $-1,35 \mathrm{c}$ & $-0,66 \mathrm{~b}$ & $0,55 \mathrm{a}$ & $0,55 \mathrm{a}$ & $0,14 \mathrm{a}$ & $0,68 \mathrm{a}$ & $0,82 \mathrm{a}$ & 65,198 & $0,000 *$ \\
3. Tradición & $-0,14 \mathrm{~b}$ & $0,11 \mathrm{~b}$ & $0,38 \mathrm{ab}$ & $-0,18 \mathrm{~b}$ & $-0,46 \mathrm{bc}$ & $0,82 \mathrm{a}$ & $-1,42 \mathrm{c}$ & 34,413 & $0,000 *$ \\
4. Conveniencia & $-1,06 \mathrm{c}$ & $0,54 \mathrm{ab}$ & $-0,60 \mathrm{c}$ & $0,88 \mathrm{a}$ & $-0,87 \mathrm{c}$ & $0,39 \mathrm{ab}$ & $-0,22 \mathrm{bc}$ & 36,466 & $0,000 *$ \\
5. Planificación & $-0,28 \mathrm{ab}$ & $0,44 \mathrm{a}$ & $-0,29 \mathrm{~b}$ & $-1,48 \mathrm{c}$ & $-0,88 \mathrm{bc}$ & $0,23 \mathrm{a}$ & $0,67 \mathrm{a}$ & 30,057 & $0,000 *$ \\
\hline
\end{tabular}

* Significativo al 1\%. Letras distintas en sentido horizontal indican diferencias estadísticamente significativas según Prueba de Comparaciones T3 de Dunnett ( $\leq 0,001)$, para varianzas no homogéneas. 
tremadamente satisfechas con su alimentación $(11,4 \%)$ (tabla 2). Esta tipología tuvo puntajes relativamente bajos en los cinco componentes del FRL, pero destacó por su nivel intermedio de planificación de las comidas (tabla 3). Tuvo mayor presencia de personas que no consume carne (14,3\%) (tabla $4)$, que nunca come en restaurantes (54,3\%), casi nunca come en locales de comida rápida y que siempre compra comida preparada $(5,7 \%)$ (tabla 5).

La tipología mayoritaria (grupo 2; 30,1\%) estuvo compuesta por mayor proporción de mujeres $(62,1 \%)$, con niños menores de 5 años (25,3\%) (tabla 2). Este grupo presentó mayores valores de los componentes "conveniencia" y "planificación" (tabla 3), destacando por preferir alimentos fáciles de preparar. El grupo 2 tuvo mayor presencia de personas que consume diariamente cereales y pastas $(34,7 \%)$, una vez/ semana carne $(41,1 \%)$, dos a tres veces por semana verduras y tubérculos $(51,6 \%)$ y una vez por semana bebidas y jugos $(14,7 \%)$ (tabla 4). Predominaron aquellos que "nunca" comen en restaurantes $(41,1 \%)$, locales de comida rápida $(56,8 \%)$, no compran comida preparada (55,8\%), ni comen en puestos ambulantes $(73,7 \%)$. Tuvo mayor presencia de personas que restringen el consumo de azúcar $(47,4 \%)$, repostería $(48,4 \%)$, sal $(48,4 \%)$; pastas, arroz o papas (26,3\%), carnes rojas $(29,5 \%)$ y café $(29,5 \%)$ (tabla 5$)$.

El grupo $3(14,6 \%)$ tuvo un alto puntaje en "vida social", pero los más bajos en "conveniencia" y "gusto por cocinar" (tabla 3). Presentó mayor proporción de personas que consumen ocasionalmente carne $(17,4 \%)$ y menor proporción que no consumen pescados y mariscos $(2,2 \%)$ y que consumen una vez por semana bebidas y jugos (1,2\%) (tabla 4). Tuvo mayor presencia de personas que casi nunca van a restaurantes $(50,0 \%)$, pero ocasionalmente van a locales de comida rápida $(73,9 \%)$ y compran comida preparada $(34,8 \%)$, con menor presencia de personas que nunca compra alimentos en puestos ambulantes (43,5\%). Fue mayor la proporción de personas que no restringe el consumo de azúcar $(80,4 \%)$, sal $(78,3 \%)$ y de pastas, arroz o papas $(97,8 \%)$ por motivos de salud (tabla 5).

El grupo $4(7,6 \%)$ estuvo conformado por mayor proporción de hombres $(79,2 \%)$, familias numerosas $(62,5 \%)$, con niños entre 5 y 13 años (45,8\%) e insatisfechos con su alimentación (41,7\%) según la SWFL (tabla 2). Esta tipología presentó un alto valor en "vida social" y "conveniencia" y la cifra más baja en "planificación" (tabla 3). En mayor proporción consumen en forma diaria cereales y pastas $(50,0 \%)$, ocasionalmente carne $(20,8 \%)$ y pescados y mariscos $(66,7 \%)$ (tabla 4). Tuvo mayor presencia de personas que ocasionalmente va a restaurantes $(54,2 \%)$, siempre $(12,5 \%)$ o generalmente $(29,2 \%)$ va a locales de comida rápida, generalmente $(16,7 \%)$ compra comida preparada y come en puestos ambulantes $(25,0 \%)$. Fue mayor la proporción de personas que no restringe el azúcar (87,5\%), los productos de repostería (87,5\%), la sal $(83,3 \%)$ y las carnes rojas $(99,9 \%)$ (tabla 5$)$.

\section{TABLA 4}

Hábitos de consumo de alimentos en el hogar (\%) de los grupos obtenidos a través de análisis de clúster, en las principales ciudades del sur de Chile, septiembre de 2010.

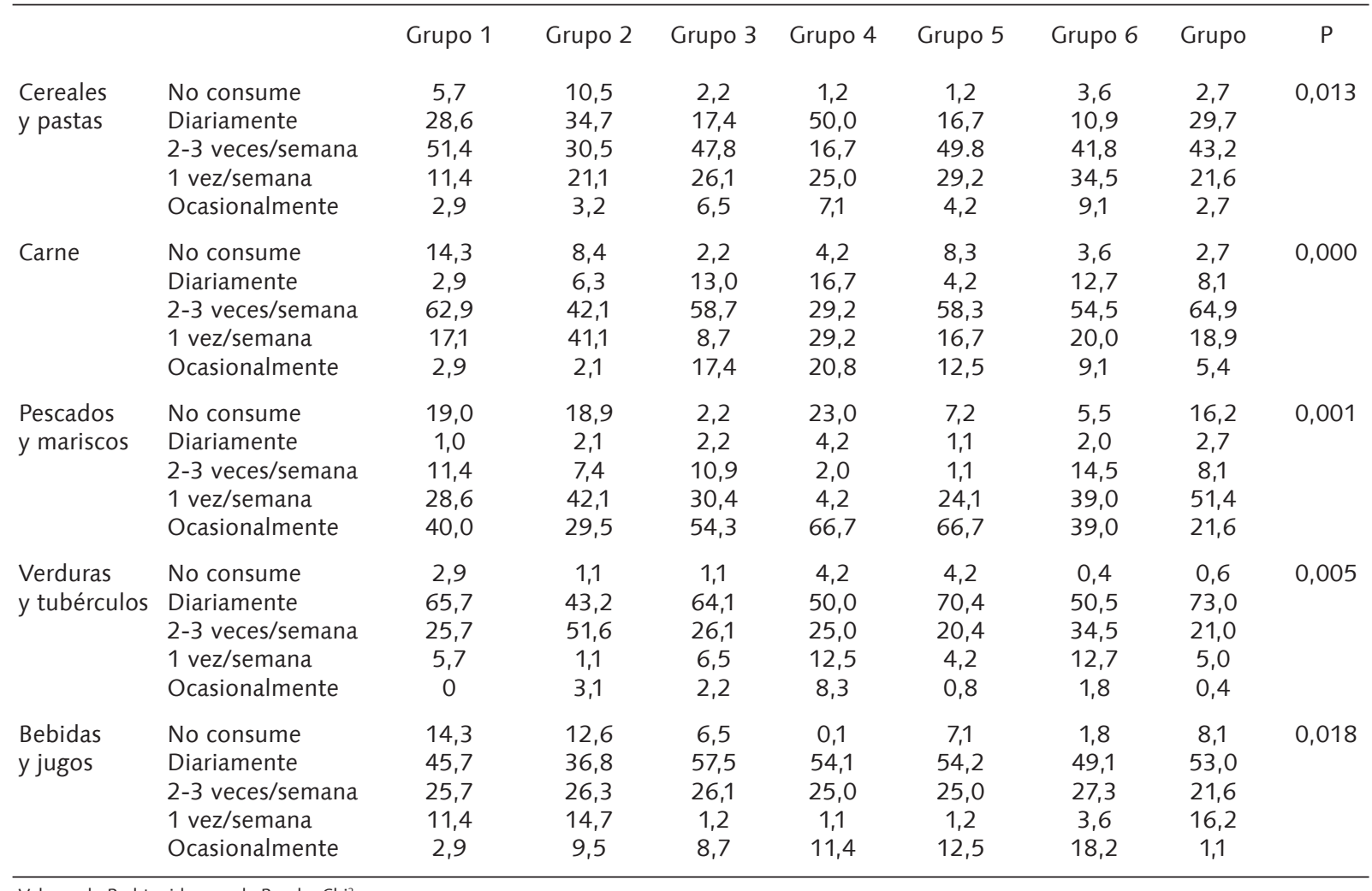

Valores de $\mathrm{P}$ obtenidos con la Prueba $\mathrm{Chi}^{2}$ 
El grupo 5 (7,6\%) estuvo formado por mayor proporción de personas con hijos entre 14 y 17 años $(41,7 \%)$ (tabla 2$)$. El grupo 5 presentó el valor más alto en el componente "gusto por cocinar" y también tuvo un valor relativamente alto en "vida social", pero en este último componente destaca por su alto grado de acuerdo con el agrado en compartir con amigos disfrutando una comida (tabla 3). Esta tipología estuvo compuesta por mayor proporción de personas que consumen ocasionalmente $(66,7 \%)$ pescados y mariscos (tabla 4$)$, generalmente comen en locales de comida rápida (33,3\%), casi nunca compran comida preparada $(50,0 \%)$ y generalmente $(25,0 \%)$ consumen alimentos de puestos ambulantes de comida (tabla 5).

La segunda tipología en tamaño (grupo 6; 17,4\%) presentó menor proporción de personas con hijos menores de 5 años $(7,3 \%)$ y una mayor proporción de personas extremadamente satisfechas con su alimentación (47,3\%) según la SWFL (tabla 2). Esta tipología tuvo altos valores en "vida" social", "tradición" y relativamente alto en "planificación", disfrutan de salir a comer fuera y de cenas con amigos en casa, pero prefieren alimentos conocidos, mantener sus hábitos alimentarios y gustan de planificar con anterioridad lo que van a comer (tabla 3). Las personas de este grupo en menor proporción consumen diariamente cereales y pastas $(10,9 \%)$ y en mayor porcentaje
$(18,2 \%)$ consumen ocasionalmente bebidas y jugos (tabla 4 ). En el grupo 6 fue mayor la proporción de personas que restringe el consumo de sal $(49,1 \%)$, pero superior la presencia de personas que no restringe el consumo de pastas, arroz o papas $(96,4 \%)$ (tabla 5). Sin embargo, esto último puede estar asociado a la menor proporción de personas que consume diariamente cereales y pastas.

El grupo $7(11,6 \%)$ presentó altos valores en "vida social" y "planificación" (tabla 3). Esta tipología consume en mayor proporción pescados una vez por semana $(51,4 \%)$ y verduras y tubérculos en forma diaria $(73,0 \%)$ (tabla 4). Fue mayor la presencia de personas que generalmente come en restaurantes $(27,0 \%)$, locales de comida rápida $(27,0 \%)$ y compra comida preparada (18,9\%) (tabla 5).

\section{DISCUSIÓN}

El presente estudio explora la existencia de distintas tipologías de personas según su estilo de vida en relación a la alimentación en el sur de Chile, mediante el uso de una adaptación del cuestionario de estilos de vida en relación a la alimentación (FRL). Mediante la aplicación de análisis cluster, fue posible distinguir siete tipologías con distinto estilo de vida en relación a su alimentación. No obstante, se optó por discutir en detalle los resultados de las tipologías más numerosas

\section{TABLA 5}

Frecuencia de comidas fuera del hogar (\%) y restricción de alimentos (\%) por motivos de salud de los grupos obtenidos a través de análisis de clúster, en las principales ciudades del sur de Chile, septiembre de 2010.

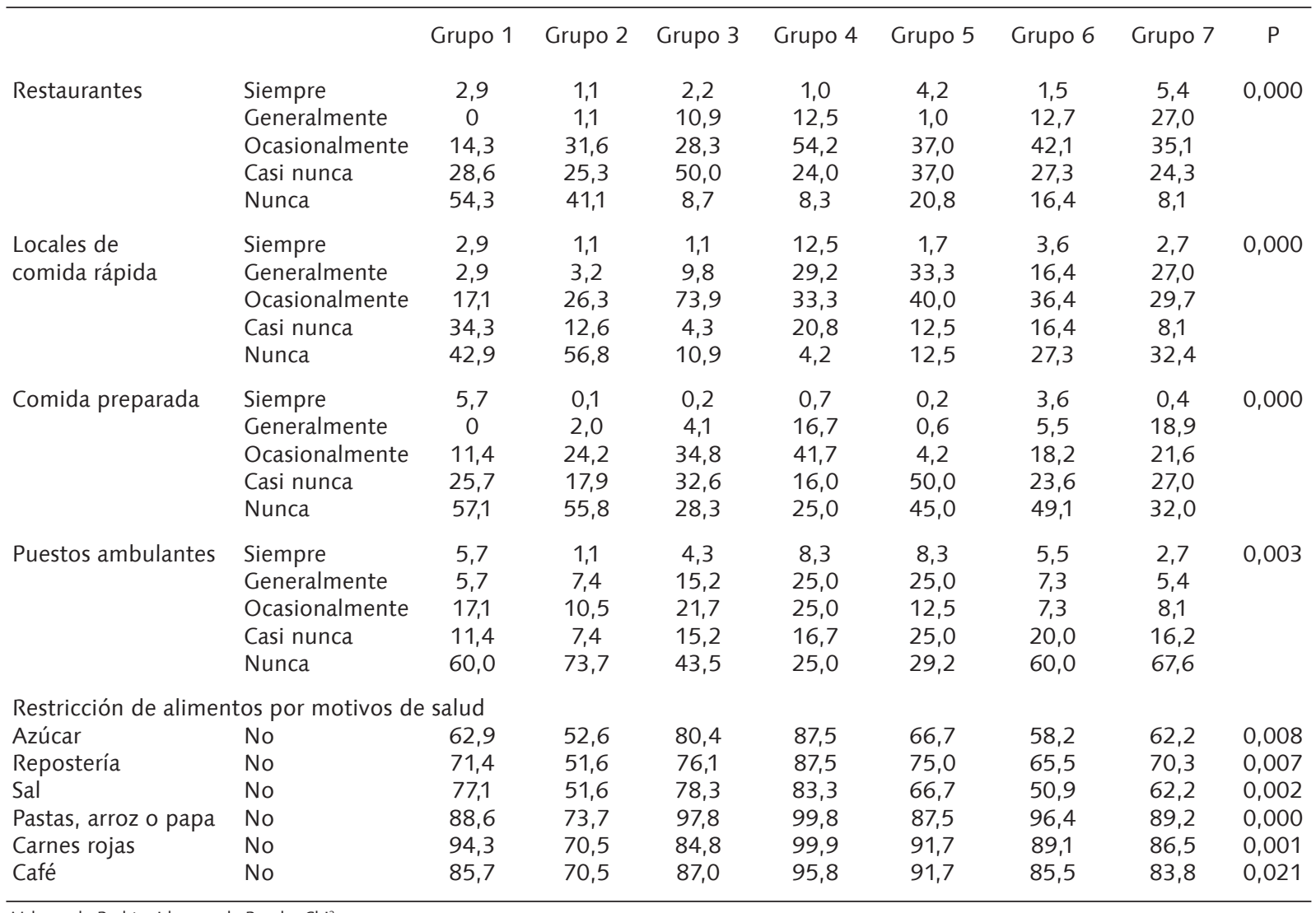

Valores de $\mathrm{P}$ obtenidos con la Prueba $\mathrm{Chi}^{2}$ 
(grupos 2, 3 y 6), pues se considera riesgoso concluir en base a grupos con bajo número de participantes (25) como ocurre en el caso de las tipologías 1, 4, 5 y $7(n=24$ - 37).

La mayor presencia de mujeres en la tipología más numerosa (grupo $2 ; 30,1 \%$ ) concuerda con la composición de género del segmento denominado "conservadores" obtenido por Wycherley et al. (14) al aplicar el FRL en Gran Bretaña. Esta característica es consistente con la menor frecuencia de consumo de carne en el grupo 2, lo que concuerda con estudios que indican una menor ingesta de este alimento en mujeres que en hombres, puesto que las mujeres tienden a preocuparse más por el cuidado de la salud y del peso (26). Esto último es congruente con la tendencia a restringir en mayor medida el consumo de alimentos asociados a enfermedades crónicas no transmisibles, no comer en locales de comida rápida ni en puestos ambulantes de comida. A la vez, denota preocupación por el cuidado de la alimentación de los niños, debido al bajo consumo de bebidas y jugos; lo que resulta incongruente con este comportamiento "saludable" en general respecto a la alimentación, es la insuficiente ingesta de verduras y tubérculos, según las sugerencias de la OMS respecto a un consumo diario de frutas y hortalizas (27). Esto puede estar asociado al bajo puntaje en el componente "gusto por cocinar" y el alto puntaje en "conveniencia", lo que se manifiesta en la preferencia por alimentos fáciles de preparar, lo que no siempre ocurre con la preparación de verduras, pero que explicaría el mayor consumo de cereales y pastas que se destacan por su fácil y rápida preparación, siendo a la vez del gusto de los niños pequeños que en esta tipología se encuentran presentes en mayor proporción. Además, existe evidencia de que madres que tratan de balancear su vida laboral con las demandas familiares, entre ellas la alimentación, optan por comidas de fácil preparación (28), siendo destacable que en esta tipología el $90,7 \%$ de las mujeres trabaja fuera del hogar. Si bien este grupo prefiere alimentos rápidos de cocinar, destaca la mayor proporción de personas que no compra comidas preparadas, posiblemente por desconfianza en la forma de preparación e ingredientes utilizados. El bajo "gusto por cocinar" y la preferencia por la "conveniencia" en la preparación de alimentos son consistentes con las características de una tipología denominada "conservadores no implicados" detectada por Nie y Zepeda (15) en Estados Unidos. Sin embargo, a diferencia de los "conservadores no implicados" en el grupo 2 se advierten numerosas conductas alimentarias saludables tendientes a prevenir enfermedades asociadas con una dieta desequilibrada.

La alta puntuación del grupo $6(17,4 \%)$ en el componente "vida social" indica que el estilo de vida en relación con la alimentación de estos participantes estaría asociado con motivos hedonistas, al disfrutar de comer en restaurantes con la familia y con los amigos, además de divertirse en cenas con amigos en casa, características similares a las encontradas por Grunert et al. (16) en una tipología denominada "interesados en la alimentación" al aplicar el FRL en China. Al respecto, los alimentos son preparados con la expectativa de ser compartidos y disfrutados en compañía, día tras día con la familia, y en los fines de semana con amigos (29), siendo congruente con el hecho de que las personas obtienen placer al comer reunidos con la familia (13). La baja puntuación de esta tipología en el componente "gusto por cocinar" es congruente con que las comidas fuera del hogar se asocian con placer y descanso de las labores de cocina, lo que se da con mayor frecuencia en parejas que no tienen hijos pequeños (29), situación que caracteriza a esta tipología. La mayor proporción de personas extremadamente satisfechas con su alimentación confirmaría que la alimentación no sólo se vincula con la salud, sino que además se asocia con la capacidad de disfrutar los alimentos y al significado hedónico que éstos tienen $(10,12)$. No obstante, en este grupo igualmente se observan conductas saludables, como el consumo ocasional de bebidas y jugos y la restricción en el consumo de sal.

La dimensión "vida social" en el grupo $3(14,6 \%)$ se manifestó principalmente por el gusto de compartir con amigos disfrutando de una comida, no así en lo relacionado a comer en restaurantes. Los bajos puntajes en "conveniencia" y "gusto por cocinar" son congruentes con la mayor frecuencia de consumo en locales de comida rápida y en puestos ambulantes $y$, de compra de comida preparada. Se ha descrito que en la medida que aumenta el consumo de alimentos fuera del hogar disminuye la calidad de la dieta (29). Esto, junto a la menor presencia de participantes que restringe el consumo de alimentos asociados a enfermedades crónicas no transmisibles y que presenta una baja frecuencia de consumo de bebidas y jugos una vez por semana, daría cuenta de hábitos alimentarios no saludables. El bajo interés por la preparación de alimentos y por la ingesta de alimentos saludables en el grupo 2, es congruente con las características del segmento denominado "consumidores descuidados" detectado por Nie y Zepeda (15) en Estados Unidos.

Si bien el presente estudio es de carácter exploratorio, fue posible identificar diferentes estilos de vida en relación a la alimentación y asociarlos con características demográficas de los participantes, sus hábitos de alimentación dentro y fuera del hogar y su nivel de satisfacción con la alimentación. El haber detectado hábitos alimentarios poco saludables sirve para el desarrollo e implementación de estrategias de intervención que propendan a fomentar hábitos de alimentación más saludables, que impacten mejorando la calidad de vida de la población. La muestra obtenida a través los criterios de inclusión en la muestra y del método de muestreo utilizado, presenta una composición similar al país en cuanto a género, zona de residencia, edad, tamaño de la familia y origen étnico (19). La principal discrepancia corresponde al nivel socioeconómico (42,1\% correspondiente al estrato $A B C 1)$, puesto que en el país la proporción de personas pertenecientes a $A B C 1$ es de aproximadamente $10 \%$ (23), constituyendo esto la principal limitación del estudio. Futuros estudios deberán abordar los estilos de vida en relación a la alimentación en muestras representativas de la realidad socioeconómica del país. Otro aspecto que deberá ser abordado es la asociación entre los estilos de vida en relación a la alimentación, la calidad de la dieta y la existencia de enfermedades crónicas no transmisibles.

\section{RESUMEN}

Con el objetivo de distinguir y caracterizar tipologías de consumidores en base a su estilo de vida en relación a la alimentación en forma exploratoria, se aplicó una encuesta a una muestra de 316 personas de las principales ciudades del sur del Chile. El instrumento de recogida de información incluyó una adaptación del cuestionario de estilos de vida en relación a la alimentación (FRL) y la escala SWFL (Satisfaction with Foodrelated Life). Mediante análisis cluster se distinguieron tres tipologías principales. La mayoritaria $(30,1 \%)$ prefiere alimentos fáciles de preparar, planifica su alimentación y mantiene sus hábitos alimentarios. La segunda (17,4\%) disfruta de salir a comer fuera y de cenas con amigos en casa, pero prefieren alimentos conocidos, son planificados y constantes en sus hábitos de alimentación. La tercera tipología (14,6\%) disfruta compartir con amigos una comida, pero no le gusta cocinar ni 
utilizar alimentos de fácil preparación. Las tipologías presentaron distinto perfil demográfico. Difirieron en la frecuencia de consumo de alimentos en el hogar y en la frecuencia de comidas fuera del hogar. Se evidenciaron diferencias en la restricción de comidas y aderezos por motivos de salud y en la satisfacción con su alimentación.

Palabras clave: Estilo de vida en relación con la alimentación, satisfacción con la alimentación, hábitos alimentarios.

Agradecimientos: Los resultados corresponden al Proyecto Fondecyt 100611.

\section{BIBLIOGRAFÍA}

1. Araneda J, Amigo H, Bustos P. Características alimentarias de adolescentes chilenas indígenas y no indígenas. Arch Latinoam Nutr 2010; 60(1): 30-5.

2. Vio F. Prevención de la obesidad en Chile. Rev Chil Nutr 2005; 32(2): 80-87.

3. Encuesta Nacional de Salud ENS Chile 2009-2010. Tomo I. Ministerio de Salud, Pontificia Universidad Católica de Chile y Universidad Alberto Hurtado, Editores. 2010. Disponible en http://www.minsal.gob.cl/portal/url/item/ bcb03d7bc28b64dfe040010165012d23.pdf [Conectado el 3 de septiembre de 2012].

4. Chiang M, Casanuevar V, Cid X, González U, Olate P, Nickel $F$, Revello L. (1999). Factores de riesgo cardiovascular en estudiantes chilenos. Salud Publica Mex 1999; 41(6): 444-51.

5. Páez M, Castaño J. Estilos de vida y salud en estudiantes de una facultad de Psicología. Psicología El Caribe 2010; 25: 155-78.

6. Flórez L. Psicología Social de la Salud. Promoción y prevención. Bogotá: Manual Moderno. 2007.

7. Lema L, Salazar I, Varela MT, Tamayo J, Rubio A, Botero A. Comportamiento y salud de los jóvenes universitarios: satisfacción con el estilo de vida. Pensamiento Psicol 2009; 5(12): 71-88.

8. Wu T, Rose S, Bancroft J. Gender differences in health risk behaviors and physical activity among middle school student. J School Nursing 2006; 22(1): 25-31.

9. Pullen C, Noble S, Fiandt K. Determinants of healthpromoting lifestyle behaviors in rural older women. Fam Community Health 2001; 24: 49-73.

10. Schnettler B, Miranda H, Sepúlveda J, Denegri M. Satisfacción con la alimentación y la vida, un estudio exploratorio en estudiantes de la Universidad de La Frontera, TemucoChile. Psico Sociedade 2011; 23(2): 426-35.

11. Schnettler B, Mora M, Miranda $H$, Sepúlveda J, Denegri $M$, Lobos $G$. Satisfacción con la alimentación en personas Mapuche en la Región de La Araucanía, Chile. Rev Chil Nutr 2012; 39(1): 18-29.

12. Dean M, Grunert K, Raats M, Nielsen NA, Lumbers M, Food in Later Life Team. The impact of personal resources and their goal relevance on satisfaction with food-related life among the elderly. Appetite 2008; 50(2-3): 308-15.

13. Brunsø K, Grunert KG. Development and testing of a cross-culturally valid instrument. Food-related lifestyle.
Adv Consumer Res 1995; 22: 475-80.

14. Wycherley A, McCarthy M, Cowan C. Speciality food orientation of food related lifestyle (FRL) segments in Great Britain. Food Qual Prefer 2008; 19: 498-510.

15. Nie C, Zepeda L. Lifestyle segmentation of US food shoppers to examine organic and local food consumption. Appetite 2011; 57: 28-37.

16. Grunert K, Perrea T, Zhou Y, Huang G, Sørensen T, Krystallis A. Is food-related lifestyle (FRL) able to reveal food consumption patterns in non-Western cultural environments? Its adaptation and application in urban China. Appetite 2011; 56: 357-67.

17. Dimech M, Caputo V, Canavari M. Attitudes for Maltese consumers towards quality in fruit and vegetables in relation to their Food-related lifestyles. Int Food Agribus Manag Rev 2011; 14(4): 21-35.

18. Pérez-Cueto F, Verbeke W, Dutra de Barcellos M, Kehagia $O$, Chryssochoidis G, Scholderer J, Grunert KG. Food-related lifestyles and their association to obesity in five European countries. Appetite 2010;54: 156-62.

19. Censo 2002. Resultados Volumen I. Población; País Región. Santiago, Chile. Instituto Nacional de Estadísticas (INE). 2003.

20. Scheaffer $R$, Mendwenhall W, Ott L. Elementos de muestreo. Ciudad de México, México: Grupo Editorial Iberoamericana S.A.; 1996.

21. Grunert K, Dean D, Raats M, Nielsen N, Lumbers M. A measure of satisfaction with food-related life. Appetite 2007; 49(2): 486-93.

22. INE. Resultados Encuestas de Presupuestos familiares. Nov. 2006-oct. 2007. Disponible en: http://www.ine.cl/canales chile_estadistico/encuestas_presupuestos_familiares/20081 resultados_EPF_2006_2007_080708a.pdf][Conectado en diciembre de 2008].

23. Adimark. Mapa socioeconómico de Chile. 2004. Disponible en http://www.adimark.cl/medios/estudios, informe_mapa_socioeconomico_de_chile.pdf] [Conectado el 20 de octubre de 2005].

24. Hair J, Anderson R, Tatham R, Black W. Análisis multivariante. Otero. $5^{a}$ ed. Madrid: Prentice Hall Internacional. Inc.; 1999.

25. McEwan JA. A comparative study of three product acceptability trials. Food Qual Prefer 1997; 8: 183-90.

26. Wyness $L$, Weichselbaum $E$, O'Connor A, Williams EB, Benelam B, Riley H, Stanner S. Red meat in the diet: an update. Nutr Bull 2011; 36: 34-77.

27. WorlD Health Organization (WHO) Study Group. Diet, nutrition and the prevention of chronic disease. WHO Technical Reports Series 797. Geneva: World Health Organization; 1990.

28. Blake C, Wethington E, Farrell T, Bisogni C, Devine C. Behavioral contexts, food-choice coping strategies, and dietary quality of a multiethnic sample of employed parents. J Am Diet Assoc 2011; 111: 401-7.

29. Kniazeva K, Venkatesh A. Food for thought: A study of food consumption in postmodern US culture. J Consum Behav 2007; 6(6): 419-35. 